\title{
Performance of Copper Calorimeter for Heat Transfer Measurement in High Enthalpy Shock Tunnel
}

\author{
WANG Qiu ${ }^{1}$, LI Jiwei ${ }^{1}$, LI Jinping ${ }^{1 *}$, ZHAO Wei ${ }^{1,2}$, JIANG Zonglin ${ }^{1,2}$
}

1. State Key Laboratory of High Temperature Gas Dynamics, Institute of Mechanics, Chinese Academy of Sciences, Beijing 100190, China

2. School of Engineering Science, University of Chinese Academy of Sciences, Beijing 100049, China

(C) SciencePress, Institute of Engineering Thermophysics, CAS and Springer-Verlag GmbH Germany, part of Springer Nature 2018

\begin{abstract}
Copper calorimeter, based on a calorimetric principle, offers a solution for heat transfer measurement in high enthalpy situation, especially in the erosive flow of high enthalpy shock tunnels. In this study, we numerically investigated the measuring performance of copper calorimeters. Non-ideal effects, such as heat loss to the insulator around and replacement of the average temperature of the copper element by the junction temperature, were discussed in detail. The influences of copper element thickness, copper/constantan wires thickness and sensor diameter were also estimated, with the aim to provide theoretical guidance for the design of copper calorimeter. In addition, corresponding experiments in JF10 high enthalpy shock tunnel were carried out against the data of coaxial thermocouples for verification. Results showed that the non-ideal thermal environment of a copper calorimeter (heat exchange with its surroundings) would result in a smaller measuring heat flux comparing to the one actually loaded; proper thickness of copper element matching the effective test time of shock tunnel was suggested. Besides, preliminary experimental results with corrections showed reasonable agreement with the heat flux of thermocouples, with an average deviation of $8 \%$. Over all, this gauge developed extends and supplements the high enthalpy shock tunnel heat transfer measurements made by other techniques.
\end{abstract}

Keywords: copper calorimeter, high enthalpy shock tunnel, heat transfer, hypersonic

\section{Introduction}

Hypersonic technology represents one of the most important issues affecting the future of the aerospace industry. High speed, shock compression, and viscous energy dissipation behind the bow shock of an aircraft head result in very high temperatures. A massive amount of kinetic energy is converted into heat energy, causing the thermal environment to become potentially critical. Transient heat transfer problems have therefore received considerable interest in numerous important applications, particularly in aerodynamic heating, where the convective surface heating rates play a major role. To this effect, accurately predicting heat transfer rates is a major issue for researchers and developers working within the current space program [1-2].

Due to the high cost of flight tests, most aerodynamic heating experiments are performed in ground facilities, where shock tunnels offer advantages in terms of being able to accommodate relatively large-size models and

Received: May 04, 2018 Corresponding author: LI Jinping, Associate Professor E-mail: lijinping@imech.ac.cn This study is financially supported by the National Natural Science Foundation of China (Grant No. 11402275, No. 11472280 and No. 11532014) and the China Scholarship Council. Associate Editor: LI Yinshi 
low operational costs. Also, the development of experimental techniques has made it possible to realize hypersonic flows ranging from 2.5 to $45 \mathrm{MJ} / \mathrm{kg}$, which correspond to velocities from 2 to $10 \mathrm{~km} / \mathrm{s}$, respectively [3-4]. In such facilities, where the effective test time is on the order of milliseconds, the heat flux rate is derived from a transient temperature monitored at selected points on the model with fast response testing technology. Generally, the techniques can be divided into two categories: one based on heat flux sensors, such as resistance thermometers [5-8], thermocouples [9-13], calorimeters [14-17], etc.; and the other based on non-intrusive techniques such as temperature sensitive paint [18-19] and thermography [20-21]. Each technique has its own benefits and challenges. For example, non-intrusive optical measurements are a candidate for obtaining global temperature distribution measurements and have temporal/spatial advantages. However, the calibration procedures are very cumbersome and the accuracy can be seriously affected by impurities of the flow field and vibration of the model. Overall, this technique remains technologically immature, especially in high enthalpy shock tunnels, where the self-luminescence of high temperature test gas exists. Because of these optical drawbacks, point heat flux sensors, typically cylindrical in shape, are primarily used for heat transfer measurements in high enthalpy facilities [22]. By the nature of the shock tunnels' operation, high enthalpy test flows can be contaminated with particulates and fragments of metallic diaphragm, particularly at high enthalpies and, even more so, at high densities [23], which will impact models at high speed, thus resulting in stringent demands on heat flux sensors. Although thin film resistance gauges can be made to have very short rise times and high electrical output per degree rise in temperature, they are prone to thermal damage and rapid erosion by small particles in the flow, and the lifetime of each gauge is limited to one or two shots [24]. Thermocouple and calorimeter are robust gauges offering a solution for high enthalpy situations. However, the junction of a thermocouple is formed either by mechanical interference or abrasion, which requires re-abrading or re-machining between shots due to damage by the erosive flow. And there are also uncertainties associated with the junction structures [25]. Instead, by using a calorimeter style gauge, the temperature sensing element can be shielded from direct contact with the erosive flows. Besides, it has the advantages of simple production, low cost and suitable for fabrication or modification according to the various requirements in the laboratory.

Calorimeter gauges are based on calculating the instantaneous heat transfer rate by measuring the time rate of the thermal energy change within a metal element, usually copper. The thermal energy is determined from a temperature measurement at the rear surface, and it is the change rate of this temperature that gives the heat flux into the exposed surface. Different types of calorimeter gauges, such as thin wall calorimeters [14], slug calorimeters [26] and null point calorimeters [27-28] have been developed by researchers to address the requirements of their special testing environments. However, each type has its own advantages and challenges. Slug and null point calorimeter gauges are generally developed for long test time measurements with relatively long response time (such as plasma wind tunnel), whereas a thin wall calorimeter is more suitable for high enthalpy shock tunnels with testing durations in the order of milliseconds. Rose [14] developed a calorimeter gauge for use in shock tubes, with testing times of up to $50 \mu$ s, using a $\sim 0.033$ $\mathrm{mm}$ thick strip of platinum as the sensing element. The operating principles and experimental setup were reported in some detail. However, this element was found to be too thin for use in high enthalpy shock tunnels with relatively long test times, where the heat flux loss to the back-up insulator could not be negligible. Taler [16] developed a mathematical technique, based on the solution of the inverse heat conduction problem, for determining the transient heat flux based on experimentally-acquired interior temperature-time data. Note that the calculations were performed by considering the classical, onedimensional heat transfer problem for a semi-infinite slab, and heat flux loss was not included.

In this study, a copper calorimeter was developed for heat transfer measurements in high enthalpy shock tunnels with a relatively thick sensitive element. The temperature distribution within the gauge was examined by solving the two-dimensional heat conduction equations numerically, thereby providing the measurement performance and correction factor simultaneously. Non-ideal effects, such as heat loss to the surrounding insulator and replacement of the average temperature of the sensitive element by the junction temperature, are also discussed in detail. Further, the gauge was verified in the JF10 high enthalpy shock tunnel through comparison to validated coaxial thermocouples and was found to achieve good performance. In all, this investigation can provide theoretical guidance for the design of a copper calorimeter and improve its measurement accuracy. This developed gauge also extends and supplements the high enthalpy shock tunnel heat transfer measurements performed by other techniques.

\section{Principle of copper calorimeter}

A schematic of our developed copper calorimeter is shown in Figure 1. It consists of a circular metal element, thermocouple wire, and epoxy insulator. The thermocouple wires (copper and constantan) are connected to the copper element by spot welding. $D$ is the sensor diameter; 
$L_{c}$ and $D_{c}$ are the thickness and diameter of the copper element, respectively. The element is attached to the insulated holder, with the objective of reducing its heat loss and enhancing robustness against erosion. In addition, the thermal junction is placed on the back of the copper, thus protecting it from the disturbances of high-velocity gas flow. The copper element first absorbs heat; its average temperature is related to the junction temperature monitored by the thermocouple on the back side. If heat loss from the copper element can be assumed to be negligible, then the net surface heat flux $q$ is obtained as follows:

$$
q=\rho c \int_{0}^{L_{c}} \frac{\partial T}{\partial t} d x=\rho c L_{c} \frac{d T_{a}}{d t}=\rho c L_{c} \frac{d T_{j}}{d t}
$$

where $\rho$ and $c$ are the density and specific heat of copper material. $T$ and $t$ are the temperature and time respectively. Subscript " $a$ " represents the average temperature of the copper element and " $j$ " the junction.

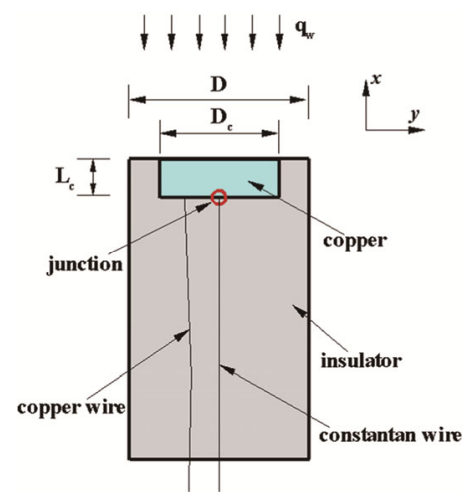

Fig. 1 Schematic diagram of copper calorimeter (not to scale)

Equation (1) is applied with several assumptions; the measurement is unfortunately complicated by the fact that any deviation (such as heat loss through the insulator and copper/constantan wire) leads to inaccuracy. In addition, the average temperature of the copper element is difficult to measure, and the key requirement is that the junction temperature gradient can be considered equal to the mean temperature gradient, with respect to time:

$$
\frac{d T_{a}}{d t}=\frac{d T_{j}}{d t}
$$

Validation of Equation (2) will be evaluated below.

\section{Simulation methodology}

The computing model in Figure 1 was simplified as an axisymmetric problem, with the copper wire replaced by a ring with the same cross-section area. The governing equations employed were axisymmetric unsteady heat conduction equations as below:

$$
\frac{\partial T}{\partial t}=\frac{k}{\rho c}\left(\frac{\partial^{2} T}{\partial x^{2}}+\frac{\partial^{2} T}{\partial y^{2}}+\frac{1}{y} \frac{\partial T}{\partial y}\right)
$$

where $x$ and $y$ are the axial and radial coordinates of the physical space; $k$ is the thermal conductivity. The material physical parameters used in the calculations are shown in Table 1, assuming constant with temperature (it is acceptable that the sensor's temperature rise is only several degrees in most cases). Equation 3 is solved by the finite difference method for spatial discretization and the fourth order Runge-Kutta method for time integration [29].

Table 1 Thermo-physical parameters of materials [30]

\begin{tabular}{cccc}
\hline Materials & $\rho / \mathrm{kg} \cdot \mathrm{m}^{-3}$ & $c / \mathrm{J} \cdot(\mathrm{kg} \cdot \mathrm{K})^{-1}$ & $\mathrm{k} / \mathrm{W} \cdot(\mathrm{m} \cdot \mathrm{K})^{-1}$ \\
\hline Copper & 8920 & 385.1 & 377.2 \\
Constantan & 8920 & 393.1 & 21.2 \\
Insulator & 1060 & 1960.0 & 0.2 \\
\hline
\end{tabular}

To improve the measuring accuracy and provide theoretical guidance for the design of copper calorimeter, its thermal environment under different parameters is examined. Detailed calculation conditions are described in Table 2. $D_{w}$ is the diameter of the copper/constantan wires. Scale of the copper calorimeter widely used in the author's laboratory is as below: $D=3.5 \mathrm{~mm}, D_{c}=3 \mathrm{~mm}$ and $L_{c}=0.15 \mathrm{~mm}$. The diameter of the copper/constantan wires is $50 \mu \mathrm{m}$ and length of the sensor is approximately $10 \mathrm{~mm}$. These are the main research objects. Structured grids of $401 \times 401$ were applied, as shown in Figure 2 . Considering that the depth of heat conduction in $x$ direction was limited during the test time (in the order of milliseconds), the computing scale along this direction was chosen to be $2 \sim 5 \mathrm{~mm}$, according to different calculation conditions. The zones near the copper element and copper/constantan wires were incorporated with clustered points. Initial temperature of the sensor was set to $T_{\infty}=293 \mathrm{~K}$ in present calculation. Since the heat transfer rate from the gas flow to the sensor was almost constant during the steady state time inherent in shock tunnels, constant heat flux $q_{w}$ was loading uniformly on its top surface, i.e., $\left(\frac{\partial T}{\partial x}\right)_{x=0}=\frac{q_{w}}{k}, t>0$. Other boundary conditions could be found in Figure 2, adiabatic boundary condition for the right and lower boundary, and symmetric boundary conditions for the left. Besides, the temperature and heat flux satisfy the continuity condition at the interface between two different materials inside the copper calorimeter.

Table 2 Calculation parameters of the calorimeter

\begin{tabular}{ccccc}
\hline Case & $L_{c} / \mathrm{mm}$ & $D / \mathrm{mm}$ & $D_{w} / \mu \mathrm{m}$ & $D_{c} / \mathrm{mm}$ \\
\hline 1 & 0.15 & 3.5 & 50 & $D-0.5$ \\
2 & $0.15,0.3,0.5$ & 3.5 & 50 & $D-0.5$ \\
3 & 0.15 & 3.5 & $50,80,100$ & $D-0.5$ \\
4 & 0.15 & $2,2.5,3.5$ & 50 & $D-0.5$ \\
\hline
\end{tabular}




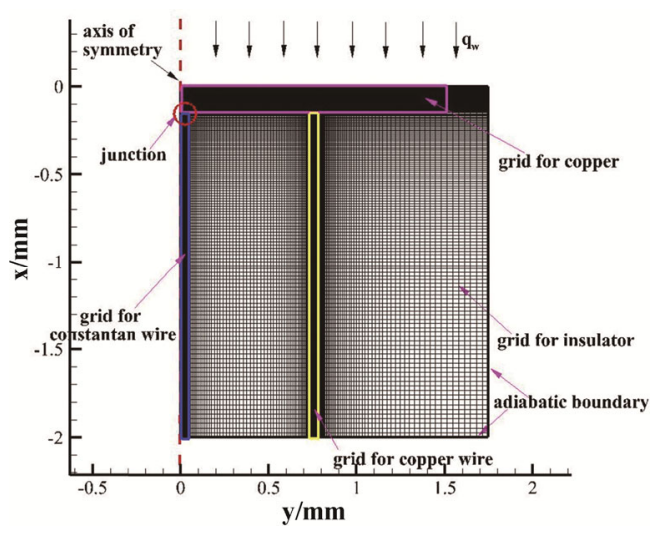

Fig. 2 Schematic diagram of computing grid

\section{Results and Discussion}

\subsection{Performance of the copper calorimeter}

Figure 3 shows the temperature field inside the copper calorimeter temperature distribution at the sensor surface or backside for Case 1 parameters at the moment of $3 \mathrm{~ms}$, with $q_{w}$ equal to $2.69 \mathrm{MW} / \mathrm{m}^{2} . \Delta T$ is the temperature increasing obtained by subtracting the initial temperature: $\Delta T=T-T_{\infty}$. It is found that temperature differences exist between the copper element and copper/constantan wires or insulator; the insulator has a much higher temperature than the copper element at the sensor surface $(x=0)$ but lower than the copper element at the sensor backside $(x=0.15 \mathrm{~mm})$, which will result in substantial heat conduction between them. This finding disagrees with the assumption of no heat loss from the copper element due to the different thermo-physical parameters of copper, insulator and constantan; the difference was especially significant for the first two materials. Overall, the copper element's thermal environment is complex; absorbing heat from the lateral insulator at the surface region, but dissipating energy to its bottom substrate. Furthermore, the temperature distributions inside the sensor also changed with time. However, this study did not particularly focus on the thermal environment in detail; instead, performance of the sensor was analyzed by comparing the heat flux monitored at the junction to the loading heat flux $\left(q_{w}\right)$, as displayed using the nondimensional form of $q_{j} / q_{w}$, where the heat transfer rate $q_{w}$ represents the loading heat flux on the sensor surface and $q_{j}$ is obtained from the junction temperature. Moreover, the results for $q_{j} / q_{w}$ could also provide the correction factors, which is especially important for the cases whose ratios are far from 1 .

Figures 4 and 5 show the temperature and heat flux curves of the junction and average of the copper element for Case 1 calculation parameters, where the horizontal axis is the time $q_{w}$ loading. Only $10 \mathrm{~ms}$ were considered, because the effective test time of a high enthalpy shock tunnel is usually shorter than this duration. As shown in Figure 4 , there is a difference between $T_{a}$ and $T_{j}$, with $\left(T_{a}-T_{j}\right) / T_{a}$ decreasing over time, with the subscript " $a$ " representing the average of the copper element and " $j$ " the junction. In other words, replacement of $T_{a}$ with $T_{j}$ is acceptable for sufficiently long time periods; although the difference is significant at the initial stage $(\sim 1 \mathrm{~ms})$ whenever $q_{w}$ is loading. Fortunately, calculation of the heat flux in Equation (1) only requires the changing rate of temperature but not the temperature itself; the heat flux is shown in Figure 5, where $q_{a}$ and $q_{j}$ are calculated as below:

$$
q_{a}=\rho c L_{c} \frac{d T_{a}}{d t}, q_{j}=\rho c L_{c} \frac{d T_{j}}{d t}
$$

The values of $q_{j} / q_{w}$ and $q_{a} / q_{w}$ are nearly identical after only $\sim 0.3 \mathrm{~ms}$, i.e., much shorter than the above described period. Therefore, it is acceptable that the junction temperature gradient can be considered equal to the mean temperature gradient, with respect to time to replace after the response time of $0.3 \mathrm{~ms}$, which demonstrates the validation of Equation (2). The values of $q / q_{w}$ in Figure 5 also suggest that the non-ideal thermal environment leads to a smaller measurement result by about $10 \%$, which can be corrected by the calculating results of $q_{j} / q_{w}$.
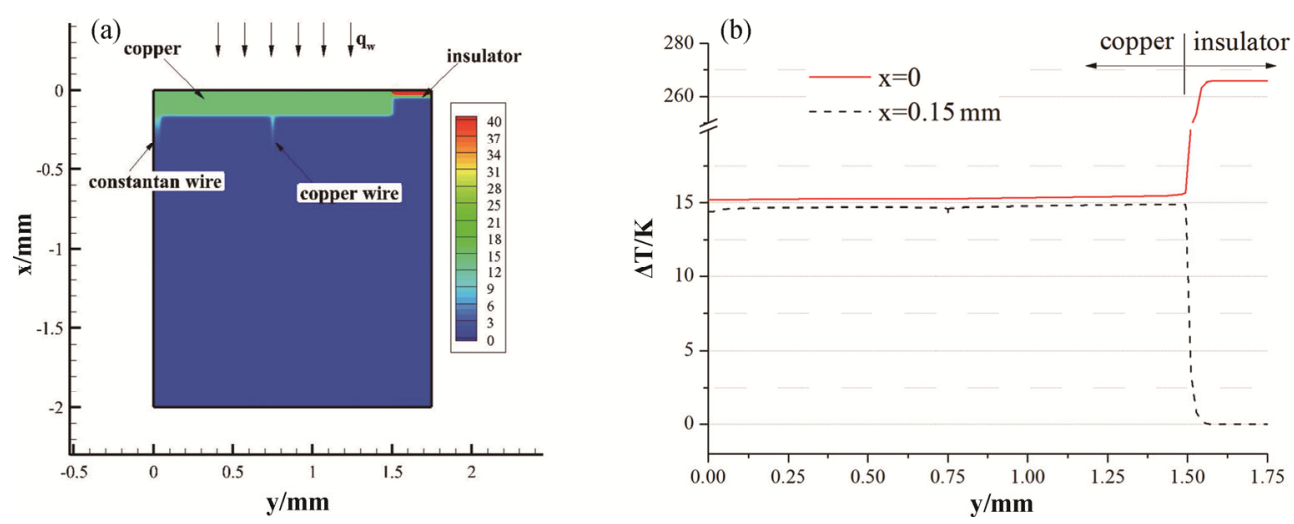

Fig. 3 Temperature distribution for Case 1 parameters at $3 \mathrm{~ms}$, (a) temperature field inside the sensor, (b) temperature distribution at the sensor surface $(x=0)$ and backside $(x=0.15 \mathrm{~mm})$ 


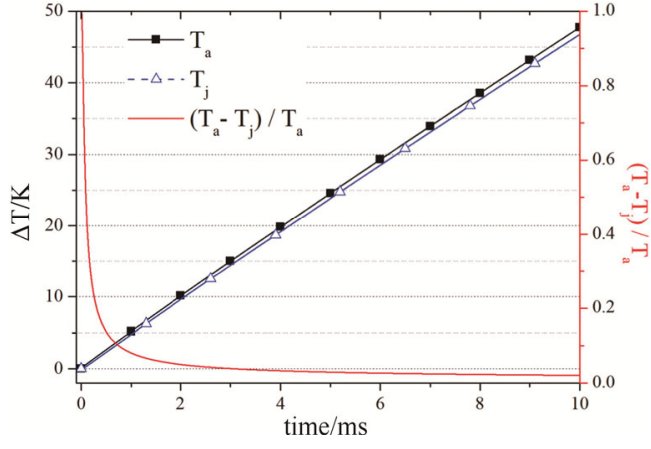

Fig. 4 Temperature curves of the junction and average of the copper element using Case 1 calculating parameters

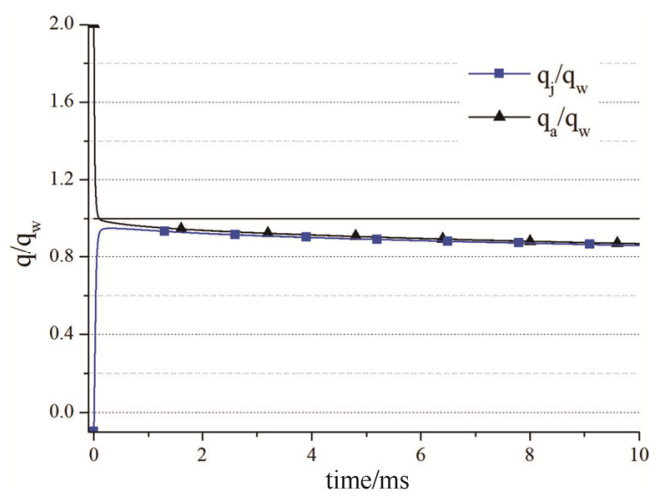

Fig. 5 Heat flux result curves of the junction and average of the copper element using Case 1 calculating parameters

The effects of copper/constantan wire diameter, copper element thickness and sensor diameter on the measurement performance of the sensor were also investigated. Figures 6 and 7 show the results from different copper element thicknesses, using Case 1 calculation parameters. Four thicknesses were tested: $0.15,0.3,0.5$ and $1 \mathrm{~mm}$. Because the response time is an important performance indicator for a heat transfer sensor, its variation with the element thickness was characterized, and the results are shown in Figure 6. The response time is defined to be the point at which $q_{j} / q_{w}=0.95$. As shown in the figure, the response time is proportional to $L_{c}$, i.e., the smaller the thickness of the copper element, the shorter the response time. At a thickness of $0.15 \mathrm{~mm}$, the response time was $0.3 \mathrm{~ms}$, which becomes $4.4 \mathrm{~ms}$ when the thickness increased to $1 \mathrm{~mm}$. However, the $1 \mathrm{~mm}$ thickness is inadvisable, for the reason that the response time is nearly the same as the effective test time of most high enthalpy shock tunnels. Figure 7 shows the influence of the copper element thickness on $q_{j} / q_{w}$, with $L_{c}=0.15,0.3$ and $0.5 \mathrm{~mm}$. It was found that the larger the thickness, the higher the value of $q_{j} / q_{w}$, and the smaller the influence of heat loss on the sensor performance. Furthermore, the values of $q_{j} / q_{w}$ continuously decrease with time increasing after the response time for the 0.15 and $0.3 \mathrm{~mm}$ cases, with the decrease being more significant for the former case. However, for a $0.5 \mathrm{~mm}$ thick sensor with a much longer response time, the proportion of heat loss to the total heat energy absorbed by the copper element is smaller, resulting in a measured heat flux that is more stable and closer to the loading heat flux. Note that the measured value, below a certain thickness, could be corrected by its $q_{j} / q_{w}$ vs. time curve, and a thicker copper element is suggested, considering a matching of its response time with the effective test time of the shock tunnel.

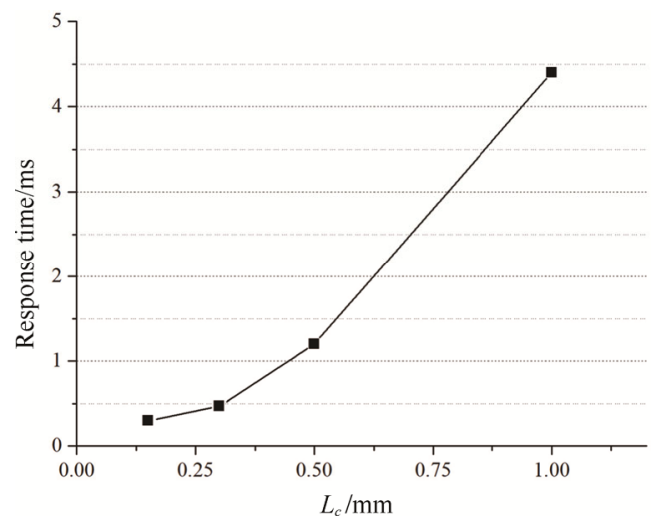

Fig. 6 Response time versus copper element thickness using Case 2 calculating parameters

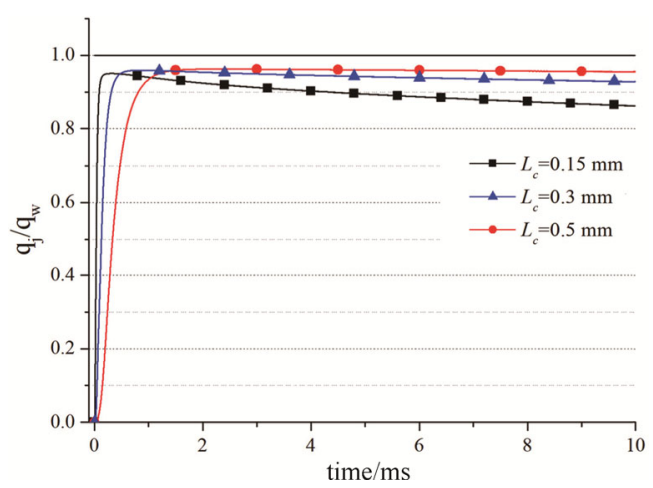

Fig. 7 Effects of copper element thickness on $q_{j} / q_{w}$ using Case 2 calculating parameters

For a copper calorimeter sensor, the thermocouple wire was soldered to the back of the copper element, which can protect it from the erosion of high velocity gas flow. Copper-constantan wires were chosen to be the thermocouple, where copper was also the material of the metal element. Unfortunately, the wires inevitably absorb some amount of heat from the copper element. Figure 8 shows the value of $q_{j} / q_{w}$ for different diameters of copper/constantan wires; the smaller the diameter, the higher the value of $q_{j} / q_{w}$, and the smaller the influence of heat loss on the sensor performance. However, the heat loss from the copper/constantan wires was not a major component, since the difference of $q_{j} / q_{w}$ between $D_{w}=50 \mu \mathrm{m}$ 
and $D_{w}=100 \mu \mathrm{m}$ was only $2 \%$. In any case, reducing the diameter of the copper/constantan wires is suggested for the design of copper calorimeters.

The sensor diameter, which is also an important parameter for conducting heat transfer measurements, affects the surface curvature, substantially affecting measuring accuracy of the sensor [31]. With the development of processing technology, thermal sensors can easily be miniaturized. However, the miniaturization of a copper calorimeter changes its inner temperature distribution, as well as the heat exchange between the lateral and bottom substrates, requiring an in-depth investigation. This study does not focus on the thermal environment in detail; instead, the value of $q_{j} / q_{w}$ with different sensor diameters is shown in Figure 9. It was found that, although $q_{j} / q_{w}$ decreases with diameter, and the heat loss influence on the

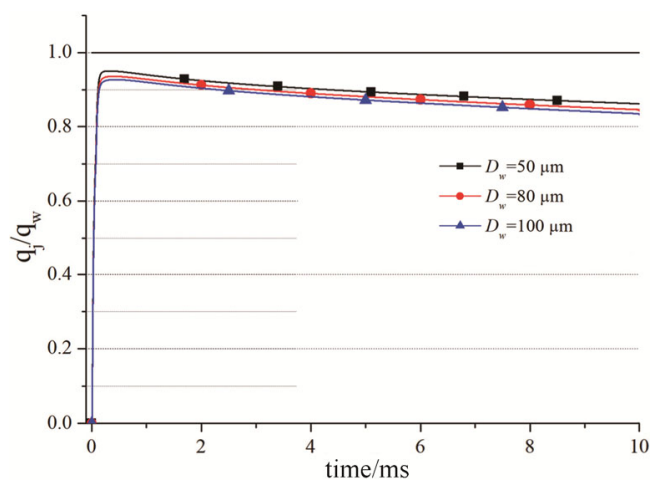

Fig. 8 Effects of copper/constantan wires diameter on $q_{j} / q_{w}$ using Case 3 calculating parameters

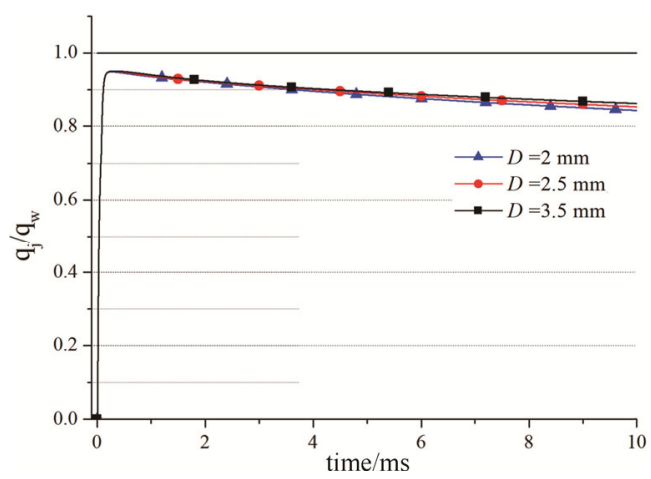

Fig. 9 Effects of sensor diameter on $q_{j} / q_{w}$ using Case 4 calculating parameters sensor performance increases, the difference in $q_{j} / q_{w}$ between different sensor diameters is almost negligible, especially for short time measurements. Thus, the proper miniaturization of a copper calorimeter is advisable for a better sensor installation.

\subsection{High enthalpy shock tunnel test results}

The above analysis provides theoretical evidence for improvement of the design of a copper calorimeter. Component analysis includes thickness of copper element optimized to match the test time, thinner copper/ constantan wires and smaller sensor diameter, under appropriate design conditions. In addition, the correction factor of a certain sensor may also be obtained using a simulation methodology. Because the copper calorimeter with the Case 1 parameters was commonly used in the author laboratory with mature technology, it has been used here for the verification of simulation methods via comparison to the heat flux of well-established E-type thermocouples, which are the rare survivable sensors in high enthalpy shock tunnels. Besides, a detailed validation of the thermocouples can be found in detail in the literature [25] within an uncertainty of $10 \%$, which is quite good for heat transfer measurements in hypersonic flows. Thus, thermocouples were chosen as the reference for the calorimeter measurements in the actual high enthalpy environment.

The experiment was conducted in the JF10 high enthalpy shock tunnel, a reflected shock tunnel using a detonation driving technique, which provides high temperature gas conditions for hypersonic flight as well as real gas effects that can also be studied. A schematic of the shock tunnel is shown in Figure 10, and consists of a detonation chamber, shock tube, nozzle and test section. A heavy metal diaphragm separates the detonation chamber and shock tube. The chamber is filled with a gaseous reactive mixture, typically oxygen and hydrogen. Strong incident shock waves in the shock tube are then easily generated by detonation product gases at high temperatures and pressures, after simultaneously igniting the reactive mixtures. Other details of the shock tunnel are available in the literatures [32-34]. Freestream parameters are listed in Table 3, with the total enthalpy of $15.2 \mathrm{MJ} / \mathrm{kg}$ and the freestream velocity of $4908 \mathrm{~m} / \mathrm{s}$.

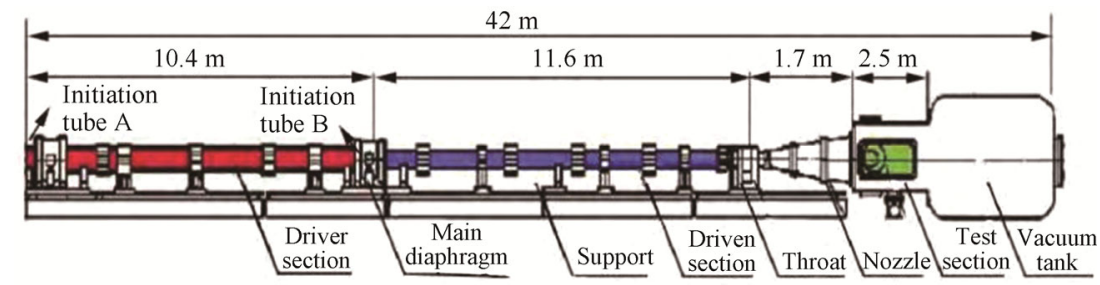

Fig. 10 Sketch of JF10 high enthalpy shock tunnel 
Table 3 Test conditions in JF10

\begin{tabular}{ccc}
\hline & Parameters & Value \\
\hline \multirow{3}{*}{ Reservoir } & $\mathrm{P}_{0} / \mathrm{MPa}$ & 14.5 \\
& $\mathrm{H}_{0} / \mathrm{MJ} \cdot \mathrm{kg}^{-1}$ & 15.2 \\
& $T_{\infty} / \mathrm{K}$ & 400 \\
& $T v_{\infty} / \mathrm{K}$ & 3182 \\
Freestream & $\rho_{\infty} / \mathrm{kg} \cdot \mathrm{m}^{-3}$ & $6.8 \times 10^{-4}$ \\
& $u_{\infty} / \mathrm{m} \cdot \mathrm{s}^{-1}$ & 4908 \\
& $p_{\infty} / \mathrm{Pa}$ & 86 \\
& $\mathrm{Re} / \mathrm{L} / \mathrm{m}^{-1}$ & $1.5 \times 10^{5}$ \\
\hline
\end{tabular}

A relatively simple configuration was selected in this study to minimize any uncertainties originating from geometric complexity. The model, a two-dimensional $7^{\circ}$ half-angle blunt wedge, is shown in Figure 11. Two rows, with each row comprising four thermal sensors, were installed in the rear flat plane, but not the blunt part, where the sensors can be installed with a relatively smooth transition with the test model. This design ensures that the accuracy of the heat transfer measurements affected by sensor installation can be optimized as much as possible. In the model, one row consisted of the copper calorimeters with Case 1 parameters and the other was $1.4 \mathrm{~mm}$ diameter E-type (chromel-constantan) coaxial thermocouples. A temperature trace of the thermocouple is shown in Figure 12. From the measured surface temperature curve, the heat flux $q$ is calculated according to Schults and Jones [14] as follows:

$$
q\left(t_{n}\right)=2 \sqrt{\frac{\rho c k}{\pi}} \sum_{i=1}^{n} \frac{T\left(t_{i}\right)-T\left(t_{i-1}\right)}{\sqrt{t_{n}-t_{i}}-\sqrt{t_{n}-t_{i-1}}}
$$

where $\rho, c$ and $k$ are the density, specific heat and thermal conductivity of the sensor material; $T$ and $t$ are the temperature and time respectively.

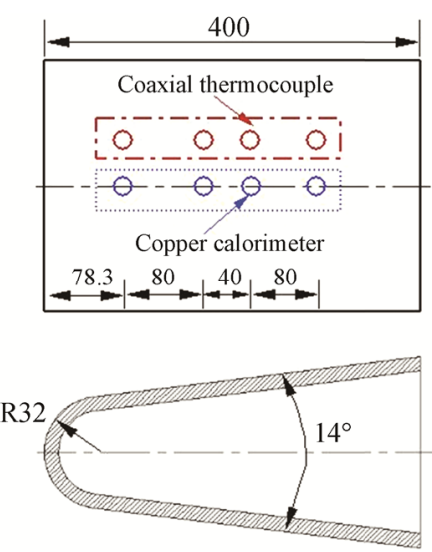

Fig. 11 Sketch of test model used for validation (not to scale)

It needs to be emphasized that both types of the sensors could survive after the experiments, though some thermocouples need to be re-abraded between shots.
Typical temperature traces of the copper calorimeter and thermocouple are shown in Figure 12. The calculated temperature curve at the junction of the copper calorimeter is also displayed, with $q_{w}$ obtained by the temperature trace of the thermocouple, that is, $0.232 \mathrm{MW} / \mathrm{m}^{2}$. Good agreement was obtained between the calculated and the experimental results for the copper calorimeter, and verification of the above simulation method is also illustrated. The effective test time of the JF10 shock tunnel under this condition is $\sim 2 \mathrm{~ms}$ after a nozzle starting time of $\sim 1.3 \mathrm{~ms}$ [35], i.e., the test period between $1.5 \mathrm{~ms}$ and $3.5 \mathrm{~ms}$ in Figure 7 and Figure 12 is seen as the effective test time, where the average value of $q_{j} / q_{w}$ is 0.91 . A correction factor of 1.1 is then used to correct the heat flux obtained by Equation (1). Results of heat flux for the four groups of copper calorimeters, with and without corrections, are shown in Figure 13, and are compared with the results from the thermocouples. It was found that the uncorrected values are lower, consistent with previous conclusions. However, the average deviation is $8 \%$ for the corrected results, which is within the $10 \%$ uncertainty of heat transfer measurements for the thermocouples. Therefore, reasonable agreement is obtained for the corrected results.

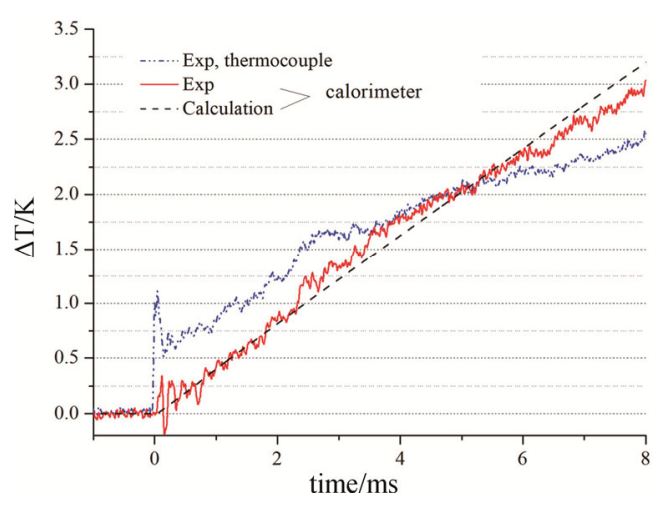

Fig. 12 Temperature traces of copper calorimeter and thermocouple

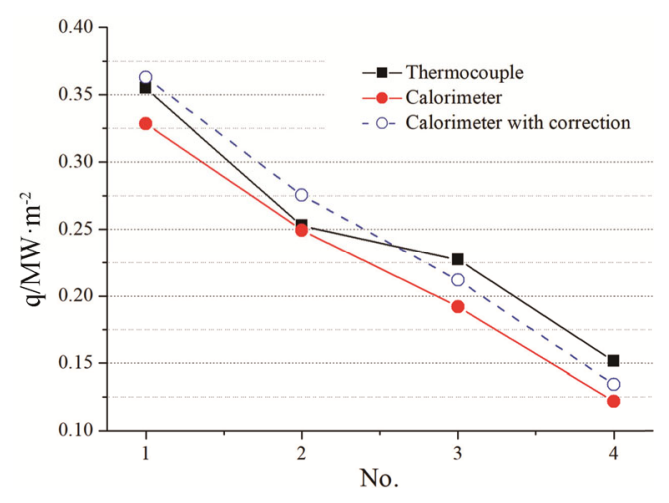

Fig. 13 Heat transfer data comparison between thermocouples and copper calorimeters 


\section{Conclusions}

In this study, we examined the measurement performance of a copper calorimeter by first solving the twodimensional heat conduction equation, and then conducting verification tests in the JF10 high enthalpy shock tunnel against the data obtained using coaxial thermocouples. From the obtained experimental and numerical results, the following conclusions were drawn. First, the non-ideal thermal environment of a copper calorimeter (heat exchange with its surroundings) results in a smaller measurement heat flux compared to the actual loaded heat flux. Secondly, the greater the thickness of the copper element, the smaller the deviation in heat flux and the longer the response time. Since the deviation is $\sim 10 \%$ for the sensor with a $0.15 \mathrm{~mm}$-thick copper element and $5 \%$ when a thickness of $0.5 \mathrm{~mm}$, a correction is suggested whenever a high-accuracy heat transfer measurement is desired. An optimization between the thickness of copper element and response time is necessary, considering the effective test time where the sensors are used. Furthermore, impacts of the sensor diameter and copper/ constantan wire diameter are found to be small, $2 \%$ at most, such that thinner wires are suggested. Finally, the experimental results of copper calorimeters in the JF10 high enthalpy shock tunnel showed reasonable agreement with the heat flux of more established E-type thermocouples, with an average deviation of $8 \%$.

Currently, work in this area is still in progress, and additional experimental studies using different sensor diameters and different thicknesses of the copper element are necessary for demonstrating and improving the construction technique. However, the preliminary results are encouraging, and the copper calorimeter gauges extend and supplement the high enthalpy shock tunnel heat transfer measurements made by other techniques.

\section{References}

[1] Qin J., Ning D.P., Feng Y., Zhang J.L., Feng S., Bao W. A new method of thermal protection by opposing jet for a hypersonic aero-heating strut. Journal of Thermal Science, 2017, 26(3): 282-288.

[2] Lu H.B, Liu W.Q. Thermal protection efficiency of forward-facing cavity and opposing jet combinational configuration. Journal of Thermal Science, 2012, 21(4): 342-347.

[3] Gai S.L., Nudford N.R. Stagnation point heat flux in hypersonic high enthalpy flows. Shock Waves, 1992, 2(1): 43-47.

[4] Hanamitsu A., Kishimoto T., Bito H. High enthalpy flow computation and experiment around the simple bodies, Tokyo: Special Publication of National Aerospace Labor- atory SP-29, 1996: 99-107.

[5] Miller C.G.I. Comparison of thin film resistance heat transfer gauges with thin-skin transient calorimeter gauges in conventional HW tunnels, NSAS Sti/recon Technical Report N, 1982.

[6] Wannenwetsch G., Ticatch L., Kidd C., Arterbury R., Measurements of wing leading edge heating rates on wind tunnel models using the thin film techniques. 20th Thermophysics Conference, Williamsburg: AIAA-19850972, 1985.

[7] Saito T., Kuribayashi T., Menzes V., Sun M., Jagadeesh G., Takayama K. Unsteady convective surface heat flux measurements on a cylinder for CFD code validation studies. Shock Waves, 2004, 13(5): 327-337.

[8] Saravanan S., Jagadeesh G., Reddy K.P.J. Convective heat transfer rate distribution over a missile shaped body flying at hypersonic speeds. Experimental Thermal and Fluid Science, 2009, 33(4): 782-790.

[9] Sanderson S.R, Sturtevant B. Transient heat flux measurement using a surface junction thermocouple. Review of Scientific Instruments, 2002, 73(7): 2781-2787.

[10] Menezes V., Bhat S. A coaxial thermocouple for shock tunnel applications. Review of Scientific Instruments, 2010, 81(10): 104905 .

[11] Mohammed H.A, Salleh H., Yusoff M.Z., Campo A. Thermal product of type-E fast response temperature sensors. Journal of Thermal Science, 2010, 19(4): 364-371.

[12] Desikan S.L.N, Suresh K., Srinivasan K., Raveendran P.G Fast response coaxial thermocouple for short duration impulse facilities. Applied Thermal Engineering, 2016, 96: 48-56.

[13] Mohammed H., Salleh H., Yusoff M.Z. Design and fabrication of coaxial surface junction thermocouples for transient heat transfer measurements. International Communications in Heat and Mass Transfer, 2008, 35(7): 853-859.

[14] Schultz D.L, Jones T.V. Heat transfer measurements in short duration hypersonic facilities, Technical Report, AGARD-AG-165, 1973.

[15] Rose P.H. Development of the calorimeter heat transfer gauge for use in shock tubes. Review of Scientific Instruments, 1958, 29(7): 557-564.

[16] Taler J. Theory of transient experimental techniques for surface heat transfer. International Journal of Heat and Mass Transfer, 1996, 39(17): 3733-3748.

[17] Wool M.R, Murphy A.J, Rindal R.A. Calorimeter measurement of heat transfer at hypersonic conditions. Journal of Spacecraft and Rockets, 1974, 11(6): 363-367.

[18] Nakakita K., Osafune T., Asai K., Global heat transfer measurement in a hypersonic shock tunnel using temperature sensitive paint. 41st AIAA Aerospace Sciences Meeting and Exhibit, Reno, Nevada, AIAA-2003-0743, 
2003.

[19] Nagai H., Ohmi S., Asai K., Nakakita K. Effect of temperature-sensitive-paint thickness on global heat transfer measurement in hypersonic flow. Journal of Thermophysics and Heat Transfer, 2008, 22(3): 373-381.

[20] Merski N.R., Reduction of analysis of phosphor thermography data with the IHEAT software package. 36th AIAA Aerospace Sciences Meeting and Exhibit, Reno, Nevada, AIAA-1998-0712, 1998.

[21] Merski N.R. Global aero-heating wind tunnel measurements using improved two-color phosphor thermography methods. Journal of Spacecraft and Rockets, 1999, 36(2): $160-170$.

[22] Wu S., Shu Y.H, Li J.P, Yu H.R. An integral heat flux sensor with high spatial and temporal resolutions. Chinese Science Bulletin, 2014, 59(27): 3484-3489.

[23] Beck W.H, Klein C., Henne U., Sachs W., Schramm J.M, Wagner A., et al. Application of temperature and pressure sensitive paints to DLR hypersonic facilities: "lessons learned". 53rd AIAA Aerospace Sciences Meeting, Kissimmee, Florida, AIAA-2015-0023, 2015.

[24] Simmons J.M. Measurement techniques in high-enthalpy hypersonic facilities. Experimental Thermal and Fluid Science, 1995, 10(4): 454-469.

[25] Li J.P, Chen H., Zhang S.Z, Zhang X.Y, Yu H.R. On the response of coaxial surface thermocouples for transient aerodynamic heating measurements. Experimental Thermal and Fluid Science, 2017, 86: 141-148.

[26] Nawaz A., Santos J.A., Assessing calorimeter evaluation methods in convective and radiative heat flux environment. 10th AIAA/ASME Joint Thermophysics and Heat Transfer Conference, Chicago, Illinois, AIAA-2010-4905,
2010.

[27] Santos J., Oishi T., Martinez E., Null point calorimeter sweeps with comparisons to thermal FEA model predictions. 41st AIAA Thermophysics Conference, San Antonio, Texas, AIAA-2009-3758, 2009.

[28] Lohle S., Battaglia J.L, Jullien P., Ootegem B.V, Lasserre J.P, Couzi J. Improvement of high heat flux measurement using a null-point calorimeter. Journal of Spacecraft and Rockets, 2008, 45(1): 76-81.

[29] Hoffmann K.A, Chiang S.T. Computational fluid dynamics, fourth ed., Wichita, Kansas: Engineering Education System, 2000.

[30] ASTM Committee E20 on Temperature Measurement. Manual on the use of thermocouples in temperature measurement, fourth ed, American Society for Testing and Materials, 1974.

[31] Zeng Z., Gui Y., Wang A.N, Qin F., Zhang H. Study on error mechanism and uncertainty assessment of heat flux measurement in shock tunnel. Journal of Experiments in Fluid Mechanics, 2015, 29(15): 15-25.

[32] Lu F.K, Marren D.E. Advanced hypersonic test facilities, AIAA: Progress in Astronautics and Aeronautics, 2002.

[33] Jiang Z.L, Yu H.R. Theories and technologies for duplicating hypersonic flight conditions for ground testing. National Science Review, 2017, 4(3): 290-296.

[34] Zhao W., Jiang Z.L, Saito T., Lin J.M, Yu H.R, Takayama K. Performance of a detonation driven shock tunnel. Shock Waves, 2004, 14(1): 53-59.

[35] Wang Q., Zhao W., Yu X.L, Jiang Z.L. Effective test time measurement research for high enthalpy shock tunnel. Acta Aeronautica et Astronautica Sinica, 2015, 36(11): 3534-353. 